\title{
ACTIVACIÓN Y PRODUCCIÓN DE SUPERFICIES SILANIZADAS
}

\section{ACTIVATION AND PRODUCTION OF SILANIZED SURFACES}

\author{
Ana Poveda G. ${ }^{*}$, Pablo Bonilla V. ${ }^{1 *}$, Joseth Rivera Q. ${ }^{1}$, \\ Javier Santamaría A. ${ }^{1}$, Lenin Ron G. ${ }^{2}$ \& Pamela Mosquera C. ${ }^{3}$
}

Recibido: 2 de junio 2020 / Aceptado: 15 de diciembre 2020 DOI: $10.26807 /$ ia.v9i1.172

Palabras claves: (7-octen-1-il) trimetoxisilano, ángulo de contacto, silanización, superficies hidrofóbicas Keywords: (7-octen-1-il) trimethoxysilane, contact angle, hydrophobic surface, silanization.

\section{RESUMEN}

En el presente trabajo se estandarizó una metodología para la activación y producción de superficies silanizadas de naturaleza hidrofóbica. La metodología fue realizada mediante la activación de grupos $\mathrm{OH}$ de superficies de vidrio eli-

1 Facultad de Ciencias Químicas, Instituto de Investigación en Salud Pública y Zoonosis-CIZ, Quito, Ecuador. (ajrivera@uce.edu.ec, jrsantamaria@uce.edu.ec, "correspondencia: apoveda@uce.edu.ec, pmbonilla@uce.edu.ec).

2 Universidad Central del Ecuador, Facultad de Ciencias Agrícolas, Quito, Ecuador. (ljron@uce.edu.ec).

3 Universidad de las Américas, Facultad de Ingeniería y Ciencias Aplicadas, Quito, Ecuador. (pmmos quera@udlanet.ec). 
minando la materia inorgánica con etanol y la materia orgánica por reacciones de oxidación, mediante la construcción de una cámara de UV/ozono con dos longitudes de onda diferentes (253,7 nm y 180 nm). Se realizó la silanización de las superficies de vidrio activadas exponiéndolas con el reactivo (7-octen1-il) trimetoxisilano/n-heptano a diferentes tiempos. La hidrofobicidad obtenida en las superficies silanizadas se determinó midiendo el ángulo de contacto $(\theta)$. Los resultados permitieron concluir que existe influencia del tiempo de exposición en la formación de superficies hidrofóbicas. Se obtuvieron ángulos de contacto de $92,417^{\circ} \pm 0,631$, tras 7 h de exposición seguidas de un proceso de limpieza de las superficies.

\section{ABSTRACT}

In this research a methodology for activation and production of silanized surfaces of hydrophobic nature was standardized. The methodology was performed by activation of $\mathrm{OH}$ groups at glass surfaces by removing inorganic matter with ethanol and organic matter by oxidation reactions in a UV / ozone chamber with two different wavelengths $(253.7 \mathrm{~nm}$ and $180 \mathrm{~nm})$. The silanization was performed by exposition of the activated surfaces to (7-octen-1-yl) trimethoxysilane / n-heptane for different times. The hydrophobicity obtained at silanized surfaces was determined by measuring the contact angle $(\theta)$. Because the data obtained it was determined that the exposure time of the reagent influences the formation of hydrophobic surfaces. After $7 \mathrm{~h}$ of exposition and cleaning of the surfaces, contact angles of $92.417^{\circ} \pm 0.631$ were obtained. 


\section{INTRODUCCIÓN}

El recubrimiento de superficies con polímeros hidrofóbicos tiene múltiples aplicaciones. Este trabajo se centra en el uso de (7-octen-1-il) trime toxisilano, para realizar una silanización o recubrimiento de superficies de vidrio. Los silanos presentan características de auto asociación y, tras su adsorción a una superficie, los grupos orgánicos de naturaleza hidrofóbica se ordenan generando una superficie hidrofóbica.

La silanización de superficies se ha utilizado para múltiples aplicaciones, entre otras, biotecnológicas de uso industrial o con fines investigativos como la inmovilización de lipasas para su reciclaje en procesos de biocatálisis (Wang et al., 2012), técnicas moleculares como el peinado de DNA (Giri et al., 2016), o la eliminación selectiva de albúmina de sueros humanos para su posterior análisis proteómico (Bhakta et al., 2017). Otras aplicaciones se han realizado en el campo de la odontología (Fornazari et al., 2017) en empastes y reparaciones por sus propiedades altamente resistentes, o para proteger materiales delicados como diato- meas (Perera, Mortazavian, \& Blum 2017).

El proceso de silanización consta de dos etapas: limpieza de la superficie y funcionalización de la superficie a silanizar. El objetivo de la etapa de limpieza es eliminar residuos orgánicos e inorgánicos y dejar expuestos los grupos - $\mathrm{OH}$ de la estructura molecular del vidrio, obteniendo una superficie activada que permita posteriormente la reacción de silanización. Diferentes métodos de limpieza de superficie de vidrio han sido reportados, siendo la limpieza con ácidos uno de los métodos más accesibles por su bajo costo (Ej. solución "piraña" $\left[\mathrm{H}_{2} \mathrm{SO}_{4}(3): \mathrm{H}_{2} \mathrm{O}_{2}(1)\right.$, $(\mathrm{v}: \mathrm{v})])$. Sin embargo, tiene como principales limitantes la alta producción de desechos contaminantes y un alto riesgo para el usuario (Labit et al., 2010). Se han desarrollado equipos que generan un plasma de barrera dieléctrica, que permiten limpiar superficies de una manera más segura para el usuario (Sung \& Hay 2014). Estos equipos requieren el uso de gases como argón, oxígeno, nitrógeno-hidrógeno, y se generan radica- 
les de oxígeno que reaccionan con la materia orgánica. Estos equipos son muy adecuados cuando la superficie a limpiar es irregular, por ejemplo, superficies microporosas, si bien la limitante es que se requiere una inversión elevada para la adquisición del equipo y de los gases. Otra alternativa económicamente más accesible es el método de limpieza empleando luz UV en combinación con ozono $\left(\mathrm{O}_{3}\right)$. Este método fue reportado por Vig (1985) utilizando un dispositivo de elaboración casera. Desde entonces, equipos de características similares pueden adquirirse en el merca do (Schwob, Renty, Coulon, Gostan, Boyer, Camet-Gabut \& Amato 2009), pero también requieren de una inversión inicial relativamente costosa. La luz UV de 253,7 y 180 nm de longitud de onda $(\lambda)$ interacciona con el $\mathrm{O}_{2}$ para generar $\mathrm{O}_{3}$ y oxígeno atómico. A su vez, estos interaccionan con la materia orgánica destruyéndola.

Una vez obtenidas superficies libres de residuos, se procede a funcionalizar la superficie con (7 octen 1 il)trimetoxisilano. El proceso de silanización puede realizarse en fase gaseosa o en fase líquida. La silanización en fase gaseosa es muy efectiva y reproducible, sin embargo, la metodología es compleja y requiere de cámaras anhidras de difícil manejo. Otra alternativa más sencilla es la silanización en fase líquida (Labit et al., 2010). Este proceso permite que una monoo multicapa de moléculas de silano se adhiera a la superficie de vidrio en presencia de un solvente como el nheptano (Figura 1A).

En este trabajo se adaptó y desarrolló un método sencillo y asequible para limpiar y silanizar superficies de vidrio, que no depende de dispositivos comerciales que implican un elevado costo. La materia inorgánica y orgánica se eliminó con solventes e irradiación UV en un dispositivo de vidrio que puede ser cargado con oxígeno (Vig, 1985). Finalmente, se realizó el proceso de silanización por reacción en líquido (Labit et al., 2010). Además, se introdujo una metodología para evaluar cuantitativamente la calidad de las superficies hidrofóbicas obtenidas, basada en la determinación del ángulo de contacto ( $\lambda$ ) (Al-Bakri et al., 2014). 
A.

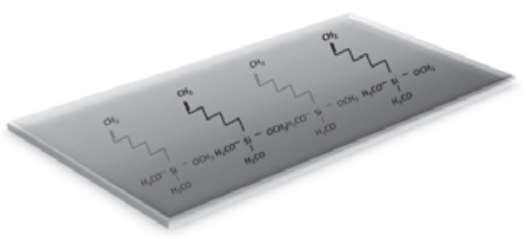

B.

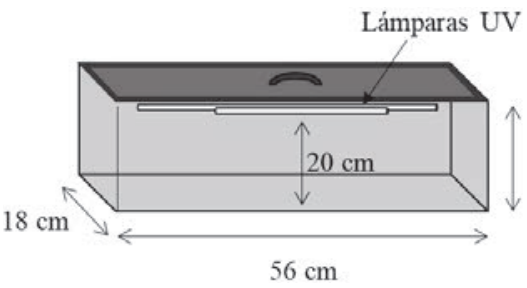

Figura 1. Representación esquemática de moléculas de (7-octen-1-il) trimetoxisilano adheridas y ordenadas sobre una superficie de vidrio, generando una superficie hidrofóbica (A).

Esquema y dimensiones de la cámara UV construida en este trabajo (B)

\section{MATERIALES Y MÉTODOS}

\section{Limpieza de superficies de vidrio}

Se utilizaron cubreobjetos $(22 \times 22$ $\mathrm{mm}$ ) como superficies de vidrio. Para eliminar la materia inorgánica y parte de la orgánica se realizó una limpieza con agua jabonosa durante 2 minutos con agitación suave. Los restos de jabón se eliminaron lavando el cubreobjetos 2 veces con $50 \mathrm{~mL}$ de agua destilada, 2 minutos. A continuación, se lavó con $20 \mathrm{~mL}$ de etanol absoluto (99 \%, Scharlau, ACS basic, ref.ET0023025P), 2 minutos y final- mente se dejó secar al aire. Los cubreobjetos se introdujeron en una cámara de UV, construida en vidrio y con dos lámparas UV de 180 nm (8 W) y una de 253,7 nm (15 W, G15t8) (Figura 1B). La cámara dispone de una salida por la cual se puede controlar la entrada y salida de gases. Con una bomba de vacío se extrajo el aire y se llenó la cámara con 1000 $\mathrm{cm}^{3}$ de oxígeno industrial. Se encendieron las lámparas y se irradió durante 3 horas a temperatura ambiente (18-20 $\left.{ }^{\circ} \mathrm{C}\right)$. 


\section{Silanización en fase líquida}

Se preparó una solución de (7-octen1-il) trimetoxisilano (grado técnico, 80 \%; Sigma Aldrich, ref. 452815) al 0,00399 M, en n-heptano (anhidro, 99 \%; Sigma Aldrich, ref. 246654) y se incubaron los cubreobjetos tras el proceso de limpieza y seleccionados al azar, durante 2-24 horas en un desecador dentro de una campana extractora. Transcurrido el tiempo los cubreobjetos se secaron al aire.

Para eliminar el exceso de silano adherido se colocaron los cubreobjetos, de uno en uno, en $20 \mathrm{~mL}$ de n-heptano y se sometió a ultrasonicación (5 min, pulsos $30 \mathrm{~s}$, al $33 \%$, con sonda microtip de $6 \mathrm{~mm}$, que equivale a una intensidad sónica de 89 $\mathrm{W} / \mathrm{cm}^{2}$ ). Se utilizó un procesador de ultrasonidos para líquidos de $130 \mathrm{~W}$ y de frecuencia $20 \mathrm{kHz}$ (modelo GEX130). Se repitió el proceso en 20 $\mathrm{mL}$ de cloroformo y se secaron los cubreobjetos al aire. Finalmente se almacenaron en un desecador.

\section{Determinación del ángulo de con- tacto $(\lambda)$}

Se determinó el ángulo de contacto utilizando la técnica goniométrica de gota yacente o sessile drop (Al-bakri et al., 2014). Para ello se depositaron $5 \mu \mathrm{L}$ de agua bidestilada sobre la superficie del cubreobjetos a analizar. Se tomó una fotografía de la gota con el objetivo 1.6X. Las imágenes se analizaron con el Image), (Schneider, C.A., Rasband, W.S., Eliceiri, 2012). Se midieron los ángulos izquierdo y derecho de cada muestra y como controles se utilizaron superficies silanizadas proporcionadas por un laboratorio (comunicación personal, (Schwob, Renty, Coulon, Gostan, Boyer, Camet-Gabut, \& Amato 2009)), superficies no tratadas y superficies silanizadas durante 7 horas sin limpieza previa. Cuanto mayor es el ángulo de contacto obtenido, mayor es la hidrofobicidad.

\section{Análisis estadístico}

Para la comparación del ángulo $(\lambda)$ de lectura generado a diversos niveles de tiempo y según el tipo de lámpara, se empleó un esquema factorial con cinco niveles de tiempo y dos tipos de lámpara. Un ANOVA (Análisis de varianza) fue establecido para las comparaciones de medias de los efectos individuales y de posible in- 
teracción. En el caso de la optimización del tiempo de medición un esquema de ANOVA de una vía fue establecido. Cuando se encontraron diferencias dentro de los efectos individuales fue aplicada la prueba de
Tukey para contrastes múltiples. La significación estadística para este estudio se estableció en el 5 \%. Para generar los gráficos se utilizó el paquete ggplot2 del software estadístico $\mathrm{R}$ versión 3.3.2 (Team, 2014).

\section{RESULTADOS}

Influencia del tiempo de exposición a silano y de la $\lambda$ de la luz $U V$, sobre la formación de superficies hidrofóbicas en matrices de vidrio

Con el objetivo de determinar si el tiempo de exposición influye sobre la calidad de la superficie hidrofóbica, se silanizaron cubreobjetos de vidrio sometiéndolos a 2, 7, 12, 17 y 24 h de exposición a (7-octen-1-il)trimeto- xisilano en fase líquida. Se realizaron 4 repeticiones cada tiempo de exposición siguiendo la metodología descrita, con la única excepción de que se utilizó únicamente una lámpara de 253,7 nm (15 W) en la cámara de UV. La hidrofobicidad de las superficies obtenidas se determinó midiendo el ángulo de contacto (Tabla $1)$.

\section{Tabla 1. Ángulos de contacto $(\theta)$ obtenidos de superficies de vidrio silanizadas previamente sometidas a irradiación UV con lámpara de $\lambda 253,7 \mathrm{~nm}$ (15 W), a diferentes tiempos. La tabla muestra los promedios (Prom), desviación estándar (SD) y la varianza}

\begin{tabular}{lccccc}
\hline & $\mathbf{2 h}$ & $\mathbf{7 h}$ & $\mathbf{1 2} \mathbf{h}$ & $\mathbf{1 7} \mathbf{h}$ & $\mathbf{2 4} \mathbf{h}$ \\
\hline Prom & 61,724 & 89,681 & 77,723 & 80,237 & 79,073 \\
+ SD & $\pm 0,851$ & $\pm 0,891$ & $\pm 0,778$ & $\pm 0,802$ & $\pm 0,867$ \\
\hline Varianza & 0,724 & 0,794 & 0,605 & 0,643 & 0,751 \\
\hline
\end{tabular}

Los resultados muestran que el mayor grado de hidrofobicidad de las superficies de vidrio silanizadas se obtuvo a las 7 horas de exposición (ángulo de contacto promedio de $89,681^{\circ} \pm$ 0,891 ), mientras que a tiempos supe- 
riores se obtienen ángulos de contacto aproximadamente $10^{\circ}$ menores (Figura 2) lo que puede deberse a un proceso de saturación de los sitios activados. Por otro lado, nos planteamos si utilizando simultáneamente lámparas de UV de 253,7 y 180 nm, podíamos obtener mejores resultados que utilizando únicamente la lámpara de UV de 253,7 nm, ya que los equipos comerciales están dotados de lámparas de ambas $\lambda$ (UV Ozone cleaner, Novascan). Para ello se instalaron dos lámparas adicionales de 180 nm (8 W) en la cámara de UV. La introducción de la $\lambda$ a $180 \mathrm{~nm}$ supone la generación de oxígeno atómico que contribuye a mejorar la eliminación de residuos orgánicos. Se repitió el experimento anterior, pero utilizando luz UV a ambas longitudes de onda: 253,7 y 180 nm, simultáneamente. Los resultados obtenidos (Tabla 2) muestran una tendencia similar a los resultados obtenidos irradiando únicamente con UV de 253,7 $\mathrm{nm}$, pero los ángulos de contacto mejoraron de $2^{\circ}$ a $3^{\circ}$ en todos los tiempos de exposición (Figura 2).

Tabla 2. Ángulos de contacto $(\theta)$ obtenidos de superficies de vidrio silanizadas previamente sometidas a irradiación UV con una lámpara de $\lambda 253,7 \mathrm{~nm}$ (15 W) y dos de $\lambda 180 \mathrm{~nm}(8 \mathrm{~W})$, a diferentes tiempos.

La tabla muestra los promedios (Prom), desviación estándar (SD) y la varianza

\begin{tabular}{lccccc}
\hline & $\mathbf{2 h}$ & $\mathbf{7 h}$ & $\mathbf{1 2} \mathbf{h}$ & $\mathbf{1 7} \mathbf{~}$ & $\mathbf{2 4} \mathbf{~}$ \\
\hline Prom & 64,215 & 92,448 & 80,082 & 81,795 & 80,559 \\
+ SD & $\pm 0,544$ & $\pm 0,497$ & $\pm 0,577$ & $\pm 0,609$ & $\pm 0,572$ \\
\hline Varianza & 0,296 & 0,247 & 0,333 & 0,371 & 0,327 \\
\hline
\end{tabular}

Estos resultados permiten concluir que la irradiación UV simultánea con $\lambda 253,7 \mathrm{~nm}$ y $\lambda 180 \mathrm{~nm}$ mejora la hidrofobicidad de las superficies obte- nidas, por lo que de aquí en adelante se trabajó con estas condiciones. Además, corroboran que el mejor tiempo de silanización son 7 horas. 


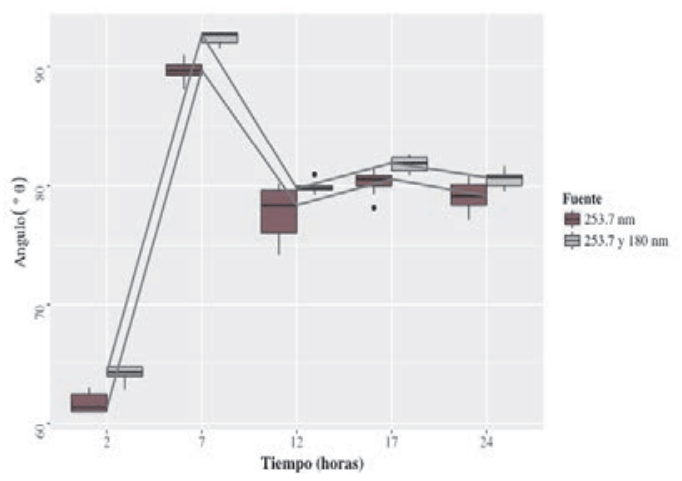

Figura 2. Tendencia obtenida en los ángulos de contacto $(\theta)$ promedio $(2,7,12,17$ y $24 \mathrm{~h})$

utilizando luz UV a $\lambda 253,7 \mathrm{~nm}$ (magenta) o utilizando $\lambda 253,7$ y $180 \mathrm{~nm}$ (gris)

Tabla 3. Análisis estadístico

ANOVA para medias clasificadas por lámpara, tiempo e interacción

\begin{tabular}{lcccccc}
\hline Fuente & gl & SC & CM & Valor F & Pr (>F) & \\
\hline Lámp. & 1,0 & 87,4 & 87,4 & 71,5 & 0,00 & $* * *$ \\
Tiempo & 4,0 & 6511 & 1627,8 & 1332,1 & $<2,2 \mathrm{e}-16$ & $* * *$ \\
Interac. & 4,0 & 5,1 & 1,3 & 1,0 & 0,39 & n.s. \\
Residual & 70,0 & 85,5 & 1,2 & & & \\
\hline
\end{tabular}

Tukey $5 \%$ de significación para Lámpara

\begin{tabular}{lcc}
\hline Grupos & Lámpara $(\boldsymbol{\lambda}, \mathrm{nm})$ & Prom. \\
\hline A & $253,7 / 180$ & 79,78 \\
B & 253,7 & 77,69 \\
\hline
\end{tabular}

Tukey $5 \%$ de significación para Tiempo

\begin{tabular}{lcc}
\hline Grupos & Tiempo (h) & Prom. \\
\hline A & 7 & 91,06 \\
B & 17 & 81,02 \\
C & 24 & 79,82 \\
D & 12 & 78,80 \\
E & 2 & 62,97 \\
\hline
\end{tabular}


Se realizó un análisis estadístico (ANOVA y prueba de Tukey, Tabla 3) que corrobora que los resultados obtenidos a tiempo 7 horas es estadísticamente diferente de los otros tiempos de exposición; igualmente los datos obtenidos a 253,7 nm son estadísticamente diferentes a los obtenidos a 253,7 y $180 \mathrm{~nm}$.

Influencia de la limpieza posterior al proceso de silanización sobre la formación de superficies hidrofóbicas sobre matrices de vidrio

Se pudo observar al microscopio que los cubreobjetos silanizados presentaban acumulaciones de residuos de (7-octen-1-il)trimetoxisilano, lo que sugería que la superficie no era homogénea. Para determinar si un proceso de limpieza tras la silanización mejora la calidad de la superficie, se realizó un ensayo con un proceso de silanización de 7 horas y se comparó con los resultados obtenidos con el mismo ensayo, seguido de un proceso de limpieza con heptano y cloroformo. Los ángulos de contacto (Tabla 4), muestran que la limpieza de posterior al proceso de silanización incrementa el promedio del ángulo de contacto en $2,7^{\circ}$, indicando que tienen una mayor hidrofobicidad. El análisis estadístico soporta que esta diferencia es significativa $y$, por tanto, se recomienda incluir el paso de limpieza posterior a la silanización en el protocolo.

Tabla 4. Ángulos de contacto $(\theta)$ obtenidos de superficies de vidrio, sin y con limpieza post-silanización. La tabla muestra los promedios (Prom), desviación estándar (SD) y la varianza obtenida en cada población

\begin{tabular}{ccc}
\hline & $\begin{array}{c}\text { Sin } \\
\text { limpieza } \\
\text { post- } \\
\text { silanización }\end{array}$ & $\begin{array}{c}\text { Con } \\
\text { limpieza } \\
\text { post- } \\
\text { silanización }\end{array}$ \\
\hline Prom & 89,860 & 92,417 \\
+ SD & $\pm 0,443$ & $\pm 0,631$ \\
\hline Varianza & 0,196 & 0,398 \\
\hline
\end{tabular}

\section{DISCUSIÓN}

Utilizando las condiciones de trabajo estandarizadas descritas en este trabajo, se obtuvieron superficies con un ángulo de contacto de 92,417 ${ }^{\circ}$, bastante próximo al ángulo de contacto obtenido en superficies adquiridas a un laboratorio externo, de $93,031^{\circ}$, Figura 3. 
Los resultados indican que la eliminación de residuos orgánicos, combinando luz UV de $\lambda$ de 253,7 y 180 nm simultáneamente, mejora la hidrofobicidad de las superficies obtenidas en aproximadamente $3^{\circ}$ (Tablas 1 y 2, 7 horas de exposición). Finalmente es recomendable eliminar el exceso de silano con la ayuda de ultrasonidos en presencia de n-heptano/cloroformo. Estas condiciones de trabajo permiten obtener superficies hidrofóbicas con un ángulo de contacto $(\lambda)$ superior a $90^{\circ}$.

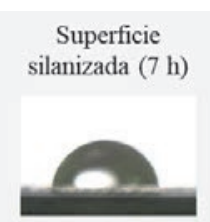

( $\theta) 92,417^{\circ}$

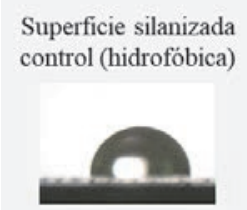

( $\theta$ ) $93,031^{\circ}$

Superficie no tratada (hidrofilica)

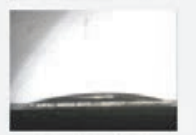

( $\theta) 19,300^{\circ}$

Figura 3. Fotografias y valores de ángulos de contacto de referencia de una gota de agua bidestilada depositada en superficie obtenida en este trabajo: superficie silanizada $7 \mathrm{~h}$, control y superficie hidrofílica. El ángulo de contacto $(\theta)$ se muestra en grados ${ }^{\circ}$

\section{CONCLUSIÓN}

En este trabajo se ha adaptado con éxito una metodología sencilla para limpiar y silanizar superficies de vidrio, independiente de dispositivos comerciales que implican un elevado costo. Los residuos de materia inorgánica y orgánica se eliminaron con solventes e irradiación UV en un dis- positivo de vidrio que puede ser cargado con oxígeno. La silanización se realizó por reacción en líquido. Finalmente, se determinó el ángulo de contacto, como método de evaluación de la hidrofobicidad de las superficies. 


\section{AGRADECIMIENTOS}

Los autores de este trabajo agradecemos la ayuda a E. S. con los cubreobjetos silanizados y a C. A. de la Escuela Politécnica Nacional por sus aportes y discusión científica. También agradecemos al personal de la Facultad de Ciencias Químicas y del Instituto de Investigación en Salud Pública y Zoonosis-CIZ por el apoyo brindado. Este trabajo ha sido financiado con recursos de la Universidad Central del Ecuador (Proyecto \#21).

\section{LISTA DE REFERENCIAS}

Al-Bakri, I., Harty, D., Al-Omari, W., Swain, M., Chrzanowski, W., \& Ellakwa, A. (2014). Surface characteristics and microbial adherence ability of modified polymethylmethacrylate by fluoridated glass fillers. Australian Dental Journal, 59(4), 482489. https://doi.org/10.1111/adj.12218

Bhakta, S., Dixit, C. K., Bist, I., Macharia, J., Shen, M., Kadimisetty, K., He, J., Dutta, B., Suib, S. L., \& Rusling, J. F. (2017). Albumin removal from human serum using surface nanopockets on silica-coated magnetic nanoparticles. Chemical Communications, 53(66), 9254-9257. https://doi.org/10.1039/C7CC03412A

Fornazari, I., Wille, I., Meda, E., Brum, R., \& Souza, E. (2017). Effect of Surface Treatment, Silane, and Universal Adhesive on Microshear Bond Strength of Nanofilled Composite Repairs. Operative Dentistry, 42(4), 367-374. https://doi.org/10.2341/16259-L

Giri, D., Li, Z., Ashraf, K. M., Collinson, M. M., \& Higgins, D. A. (2016). Molecular Combing of $\square$-DNA using Self-Propelled Water Droplets on Wettability Gradient Surfaces. ACS Applied Materials \& Interfaces, 8(36), 24265-24272. https://doi.org /10.1021/acsami.6b08607

Labit, H., Goldar, A., Guilbaud, G., Douarche, C., Hyrien, O., \& Marheineke, K. (2010). Silanization of coverslips for DNA combing. BioTechniques. https://doi.org/10. 2144/000113255 
Perera, H. J., Mortazavian, H., \& Blum, F. D. (2017). Surface Properties of Silane-Treated Diatomaceous Earth Coatings: Effect of Alkyl Chain Length. Langmuir, 33(11), 2799-2809. https://doi.org/10.1021/acs.langmuir.7b00015

Schneider, C.A., Rasband, W.S., Eliceiri, K. W. (2012). NIH Image to ImageJ: 25 years of image analysis. Nature Methods, 9, 671-675.

Schwob, E., Renty, C. de, Coulon, V., Gostan, T., Boyer, C., Camet-Gabut, L., \& Amato, C. (2009). Use of DNA Combing for Studying DNA Replication In Vivo in Yeast and Mammalian Cells. In Methods in molecular biology (Vol. 521, pp. 493-507). https://doi.org/10.1007/978-1-60327-817-5

Sung, S. H., \& Hay, M. (2014). Standard Operating Procedure Hood for O 2 Plasma Cleaner \& Spincoater Description of Process General. Retrieved from https://engineering.purdue.edu/Powerlab/Standard $\% 20$ Operating $\% 20$ Procedures $/ v$ Final $\% 20$ O2\%20Plasma\%20and\%20Spincoater\%20\%202014.pdf

R Core Team (2014). R: A language and environment for statistical computing. R Foundation for Statistical Computing. Retrieved from http://www.r-project.org/

Vig, J. R. (1985). UV/ozone cleaning of surfaces. Journal of Vacuum Science \& Technology, 3(3).

Wang, J., Meng, G., Tao, K., Feng, M., Zhao, X., Li, Z., Xu, H., Xia, D., \& Lu, J. R. (2012). Immobilization of Lipases on Alkyl Silane Modified Magnetic Nanoparticles: Effect of Alkyl Chain Length on Enzyme Activity. PLoS ONE, 7(8), 21-25. https://doi. org/10.1371/journal.pone.0043478 